\title{
Regional Metabolite Concentrations in Human Brain as Determined by Quantitative Localized Proton MRS
}

\author{
Petra J. W. Pouwels, Jens Frahm
}

\begin{abstract}
The regional distribution of brain metabolites was studied in several cortical white and gray matter areas, cerebellum, and thalamus of young adults with use of quantitative single-voxel proton MRS at 2.0 T. Whereas the neuronal compound $\mathrm{N}$ acetylaspartate is distributed homogeneously throughout the brain, $\mathrm{N}$-acetylaspartylglutamate increases caudally and exhibits higher concentrations in white matter than in gray matter. Creatine, myo-inositol, glutamate, and glutamine are less concentrated in cortical white matter than in gray matter. The highest creatine levels are found in cerebellum, parallel to the distribution of creatine kinase and energy-requiring processes in the brain. Also myo-inositol has highest concentrations in the cerebellum. Choline-containing compounds exhibit a marked regional variability with again highest concentrations in cerebellum and lowest levels and a strong caudally decreasing gradient in gray matter. The present findings neither support a metabolic gender difference (except for a 1.3-fold higher myo-inositol level in parietal white matter of female subjects) nor a metabolic hemispheric asymmetry.
\end{abstract}

Key words: proton spectroscopy; brain metabolism; cerebral metabolite distribution.

\section{INTRODUCTION}

Quantification of absolute metabolite concentrations in human brain is required not only for a better understanding of in vivo neurochemistry but also for an unambiguous assessment of pathologic alterations and pertinent applications in clinical diagnosis and therapy monitoring. Initial emphasis of localized proton MRS studies has been placed on the evaluation of metabolite patterns specific for tissue types such as gray and white matter (1-4). However, because both gray and white matter extend over large cortical and subcortical areas with distinct anatomic and functional properties, more detailed differences are likely to be expected. This hypothesis has been supported by a preliminary short-echo time proton MRS study of cortical gray matter (5). Pertinent findings are in line with earlier MRS reports on regional metabolic heterogeneity although such studies were often compromised by the use of mixed tissue volumes, long echo times, and/or ratios of resonance intensities or concentrations $(6-10)$.

The primary aim of the present work therefore was a reliable and detailed determination of absolute metabo-

\section{MRM 39:53-60 (1998)}

From the Biomedizinische NMR Forschungs GmbH am Max-Planck-Institut für biophysikalische Chemie, Göttingen, Germany.

Address correspondence to: Petra J. W. Pouwels, Ph.D., Biomedizinische NMR Forschungs GmbH am Max-Planck-Institut für biophysikalische Chemie, D-37070, Göttingen, Germany.

Received March 24, 1997; revised June 2, 1997; accepted July 6, 1997

This work was supported by a research grant from the European Union (Program Biomed II) (to P.J.W.P.).

0740-3194/98 \$3.00

Copyright 1998 by Williams \& Wilkins

All rights of reproduction in any form reserved. lite concentrations in multiple locations of cortical and. subcortical gray and white matter. In addition, the data were analyzed for putative gender differences and hemispheric asymmetry.

\section{METHODS}

Studies of 34 healthy volunteers ( 17 female/17 male, age range $18-39$ years, mean $27 \pm 4$ years) were conducted at $2.0 \mathrm{~T}$ (Siemens Magnetom SP4000, Erlangen) using the standard imaging headcoil. Informed written consent was obtained before all examinations. A total of 41 studies were performed yielding about five spectra each.

Figure 1 shows representative volumes-of-interest (VOI) selected for localized proton MRS. Cortical white matter was measured in frontal, parietal, and occipital brain (Fig. 1a) with small volumes of 4.1-6.4 $\mathrm{ml}$ to prevent contamination with gray matter. Gray matter volumes in frontal (8-12 ml), parietal (8-18 ml), and occipital brain $(8 \mathrm{ml})$ were located in paramedian positions spanning $20 \mathrm{~mm}$ across the interhemispheric fissure (Fig. $1 b)$. In addition, gray matter was investigated in insular areas $(10 \mathrm{ml})$ and thalamus $(6.4 \mathrm{ml})$ (Fig. 1c). The cerebellum ( $8 \mathrm{ml}$ ) was examined both in a central position covering the vermis (Fig. 1b) and in hemispheric locations containing mainly white matter (Fig. 1d).

Fully relaxed, short-echo time proton MR spectra were recorded with use of a single-voxel STEAM localization sequence $(T R / T E / T M=6000 / 20 / 30 \mathrm{~ms}, 64$ accumulations) as described previously (11). Particular care was taken to optimize magnetic field homogeneity and water suppression by localized adjustments. Spectroscopic time-domain data were corrected for residual eddy current effects (12) and calibrated in proportion to the actual coil loading by using the transmitter reference amplitude (3). For display purposes only, spectral postprocessing involved zero-filling to $4 \mathrm{~K}$ complex data points (2048 $\mathrm{ms}$ ), Gaussian filtering (half-width $317 \mathrm{~ms}$ ), and manual phase-correction. Such spectra were not used for quantification.

Absolute metabolite concentrations were estimated by LCModel, a user-independent fitting routine that employs a library of concentration-calibrated model spectra of all individual metabolites $(3,13)$. The method exploits the full spectroscopic information of each metabolite and not just isolated intense resonances. It therefore allows a distinction even between metabolites with overlapping signals provided they exhibit additional proton resonances at different frequencies. Because the separation of two overlapping metabolites also depends on the spectral resolution attainable in vivo, LCModel not only yields individual concentrations of such metabolites, but also offers a treatment as the sum of both. For example, total NAA (tNAA) is determined in addition to separate con- 

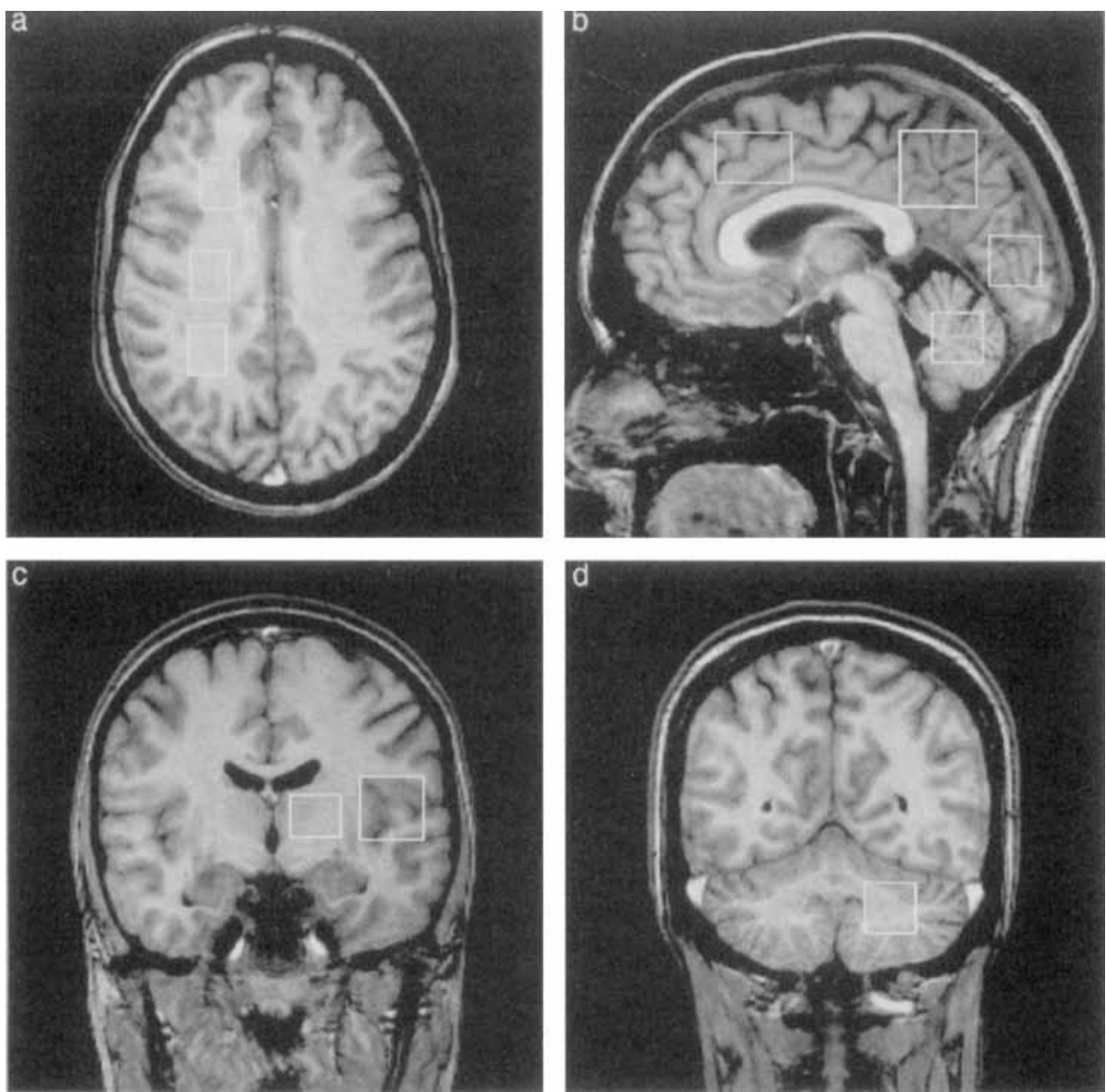

FIG. 1. $T_{1}$-weighted MR images (RF spoiled 3D FLASH, TR/TE $=15 / 6 \mathrm{~ms}$, flip angle $20^{\circ}$ ) showing representative locations selected for proton MRS of white matter in frontal, parietal, and occipital brain (a); gray matter in paramedian frontal, parietal, and occipital brain, as well as in the central cerebellum (b); gray matter in the insular area and in thalamus (c); and white matter in the cerebellar hemisphere (d).

centrations of $N$-acetylaspartate (NAA) and $N$-acetylaspartylglutamate (NAAG) (14). Similarly, the amount of choline-containing compounds (Cho) is expressed as the sum of phosphorylcholine (PCh) and glycerophosphorylcholine (GPC) as suggested by in vivo phosphorus MRS (15) and in vitro proton MRS $(16,17)$. The need for including GPC in the library of model spectra was evidenced by a discrepancy between experimental spectra and the corresponding LCModel fits, i.e., the appearance of a 3.68-ppm proton resonance in the residual "baseline," when PCh was the only Cho-compound. Most likely, this resonance belongs to the methylene protons of the glycerol backbone of GPC.

Macromolecules with short $T_{2}$ relaxation times also contribute to short echo-time spectra $(18,19)$. However, the baseline estimated by LCModel includes resonances with broad linewidths. Thus, the quantitation of small, mobile metabolites is not affected by macromolecular contributions. In this study no attempt has been made to further characterize residual broad resonances.

Concentrations are expressed as $\mathrm{mmol} /$ liter VOI (i.e., as $\mathrm{mM}$ ) and are not corrected for CSF contributions and residual $T_{2}$ relaxation effects. $T_{1}$ saturation was ignored as all experiments were performed under fully relaxed conditions using sufficiently long repetition times $T R$. Because the model spectra were measured with similar parameters as the in vivo spectra, only differences in $T_{2}$ relaxation between in vivo and in vitro must be taken into account. For $T_{2}$ relaxation times between 130 and $370 \mathrm{~ms}$ in vivo and approximately 800 $\mathrm{ms}$ in vitro correction factors range from 1.03 to 1.14 at an echo time of $T E=20 \mathrm{~ms}$ (3). However, because $T_{2}$ relaxation times of metabolites show a regional dependence $(1,4,6,7)$, such small corrections were ignored as they would be at the expense of time-consuming determinations of metabolite $T_{2}$ values in each VOI that are not tolerable for related MRS studies of patients. For similar reasons, data were not corrected for potential contributions from CSF. Pertinent correction factors amount to about 1.05-1.07 for gray matter of young healthy adults $(3,4)$, and even less for other regions. In patient studies the observation of reduced metabolite concentrations may reflect brain atrophy due to larger CSF contributions. In that case a comparison with healthy controls either may be based on concentration ratios, or requires an estimation of the CSF contribution to the VOI, e.g., by using image segmentation.

Statistical comparisons between regions were performed with two-sided unpaired $t$ tests assuming unequal variances. Gender differences were assessed by applying these tests to metabolite concentrations of female and male subjects for each region separately. Data from hemispheric regions (cortical white matter, insular gray matter, cerebellar hemispheres, and thalamus) were further examined for right-left asymmetry, both intraindividually and interindividually. The intraindividual asymmetry parameter (individual laterality) was defined as the concentration ratio of a single metabolite in right versus left hemispheres for each subject with bilateral spectral acquisitions. The interindividual asymmetry (species laterality) was assessed by comparing concentrations from right and left hemisphere of all subjects with a two-sided $t$ test.

\section{RESULTS}

\section{White Matter}

Representative proton MR spectra of frontal, parietal, and occipital white matter are shown in Fig. 2. The regional 


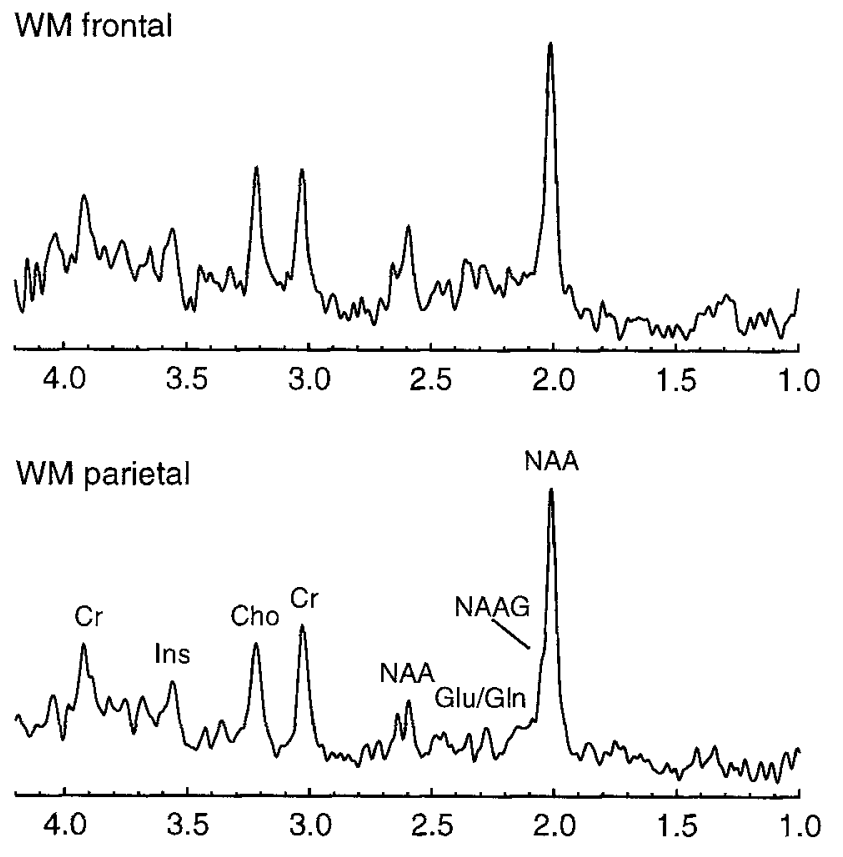

WM occipital

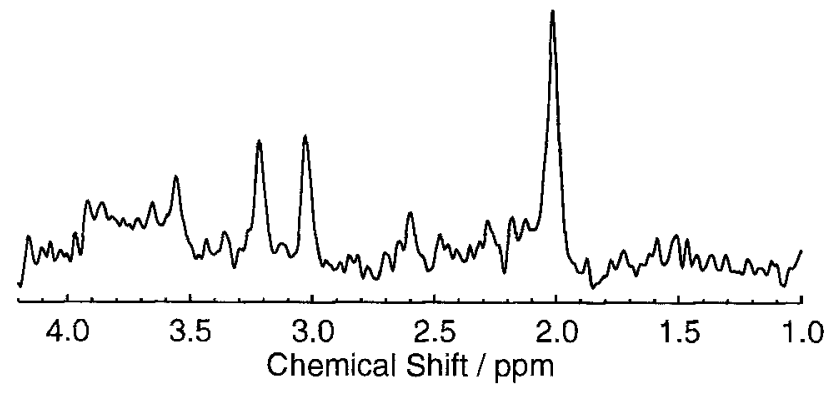

FIG. 2. Localized proton MR spectra (STEAM, TR/TE/TM $=6000 /$ $20 / 30 \mathrm{~ms}, 64$ accumulations) of a 22-year-old subject in frontal $(5.1 \mathrm{ml})$, parietal $(6.4 \mathrm{ml})$, and occipital white matter $(5.1 \mathrm{ml})$. Major metabolites refer to $\mathrm{N}$-acetylaspartate (NAA), $\mathrm{N}$-acetylaspartylglutamate (NAAG), glutamate (Glu), glutamine (Gln), total creatine (Cr), choline-containing compounds (Cho), and myo-inositol (Ins).

similarity is confirmed by an analysis of mean metabolite concentrations in white matter of all subjects summarized in Table 1. Creatine and phosphocreatine (Cr) as well as Cho are distributed uniformly throughout cortical white matter. In contrast, the concentration of tNAA is significantly lower in frontal white matter than in parietal or occipital white matter. Separation of NAA and NAAG shows that this distribution of tNAA is almost exclusively due to NAAG, whereas no significant regional differences are observed for NAA (14). Thus, the contribution of NAAG to tNAA varies from $15 \%$ in frontal white matter to $25 \%$ in parietal and occipital white matter.

A regional dependence is also observed for myo-inositol (Ins). While this metabolite is equally distributed in periventricular white matter frontally and occipitally, its concentration in parietal white matter is significantly lower. Finally, no regional variations are revealed for glutamate (Glu) and glutamine (Gln).

\section{Gray Matter}

Gray matter spectra of frontal, parietal, and occipital brain, as well as of the insular area are shown in Fig. 3. The most striking observation is a gradient in Cho yielding an intensity decrease from frontal to occipital cortex. A quantitative analysis of all gray matter spectra in Table 2 confirms highly significant differences in Cho concentration between frontal and parietal, as well as between parietal and occipital gray matter. When gray and white matter regions are compared, even the highest levels of Cho in frontal gray matter are lower than those found in white matter.

The regional variability of $\mathrm{tNAA}$, which has a higher concentration in occipital than in parietal or frontal gray matter, is due to enhanced levels of both NAA and NAAG in occipital cortex. Whereas the concentration of NAA in frontal and parietal gray matter is similar to that in white matter, it is significantly higher in occipital cortex. Between and within white and gray matter, the largest variability is observed for NAAG, which has lower concentrations in gray matter, but shows a caudal increase in both tissues (14). Cr, Ins, Glu, and Gln are distributed homogeneously throughout paramedian gray matter but at higher levels than in white matter. For all metabolites, concentrations in the insular area are higher than in parietal gray matter. Similar concentration ratios with respect to $\mathrm{Cr}$ suggest that this may partly be explained by reduced CSF contributions in insular areas versus paramedian locations.

Concentrations of other brain metabolites like scylloinositol, glucose, lactate, taurine, $\gamma$-amino butyric acid, and aspartate are also determined by LCModel. However, unfavorable conditions such as considerable spectral overlap, strong coupling, and rather low concentrations usually prevent a reliable determination in individual subjects. On the other hand, group averages result in reasonable estimates of pertinent concentrations in parietal gray matter $(n=19)$ of $0.14 \pm 0.09 \mathrm{mM}$ for scylloinositol, $0.5 \pm 0.6 \mathrm{mM}$ for glucose, $0.6 \pm 0.4 \mathrm{mM}$ for lactate, $1.0 \pm 0.4 \mathrm{mM}$ for taurine, $1.4 \pm 0.8 \mathrm{mM}$ for $\gamma$-amino butyric acid, and $1.4 \pm 0.8 \mathrm{~m} M$ for aspartate.

Table 1

Metabolite Concentrations in White Matter $(\mathrm{mM} \pm \mathrm{SD})^{\mathrm{a}}$

\begin{tabular}{|c|c|c|c|}
\hline & $\begin{array}{l}\text { Frontal } \\
\mathrm{n}=22\end{array}$ & $\begin{array}{l}\text { Parietal } \\
n=20\end{array}$ & $\begin{array}{c}\text { Occipital } \\
n=27\end{array}$ \\
\hline $\mathrm{Cr}$ & $5.7 \pm 0.5$ & $5.7 \pm 0.6$ & $5.5 \pm 0.8$ \\
\hline tNAA & $9.6 \pm 1.1^{\star *}$ & $10.6 \pm 0.8$ & $10.4 \pm 0.9$ \\
\hline NAA & $8.1 \pm 0.9$ & $8.0 \pm 1.0$ & $7.8 \pm 0.9$ \\
\hline NAAG & $1.5 \pm 0.9^{* \star *}$ & $2.7 \pm 1.2$ & $2.6 \pm 1.0$ \\
\hline Cho & $1.78 \pm 0.41$ & $1.68 \pm 0.27$ & $1.64 \pm 0.21$ \\
\hline Ins & $3.8 \pm 0.9^{*}$ & $3.1 \pm 0.6$ & $4.1 \pm 0.8^{\star \star *}$ \\
\hline Glu & $7.0 \pm 2.6$ & $6.7 \pm 1.8$ & $6.0 \pm 1.2$ \\
\hline Gln & $1.8 \pm 1.6$ & $1.5 \pm 1.3$ & $2.2 \pm 1.6$ \\
\hline $\mathrm{FWHM}^{\mathrm{b}}$ & $3.51 \pm 0.39$ & $3.45 \pm 0.31$ & $3.31 \pm 0.34$ \\
\hline
\end{tabular}

a $\mathrm{m} M$ refers to $\mathrm{mmol} /$ liter $\mathrm{VOI}$.

${ }^{b}$ FWHM: full linewidth at half maximum $(\mathrm{Hz})$ of the $\mathrm{Cr}$ signal at $3.03 \mathrm{ppm}$ ${ }^{* * *} p<0.001,{ }^{* *} p<0.01,{ }^{*} p<0.05$ for comparisons to parietal white matter. 

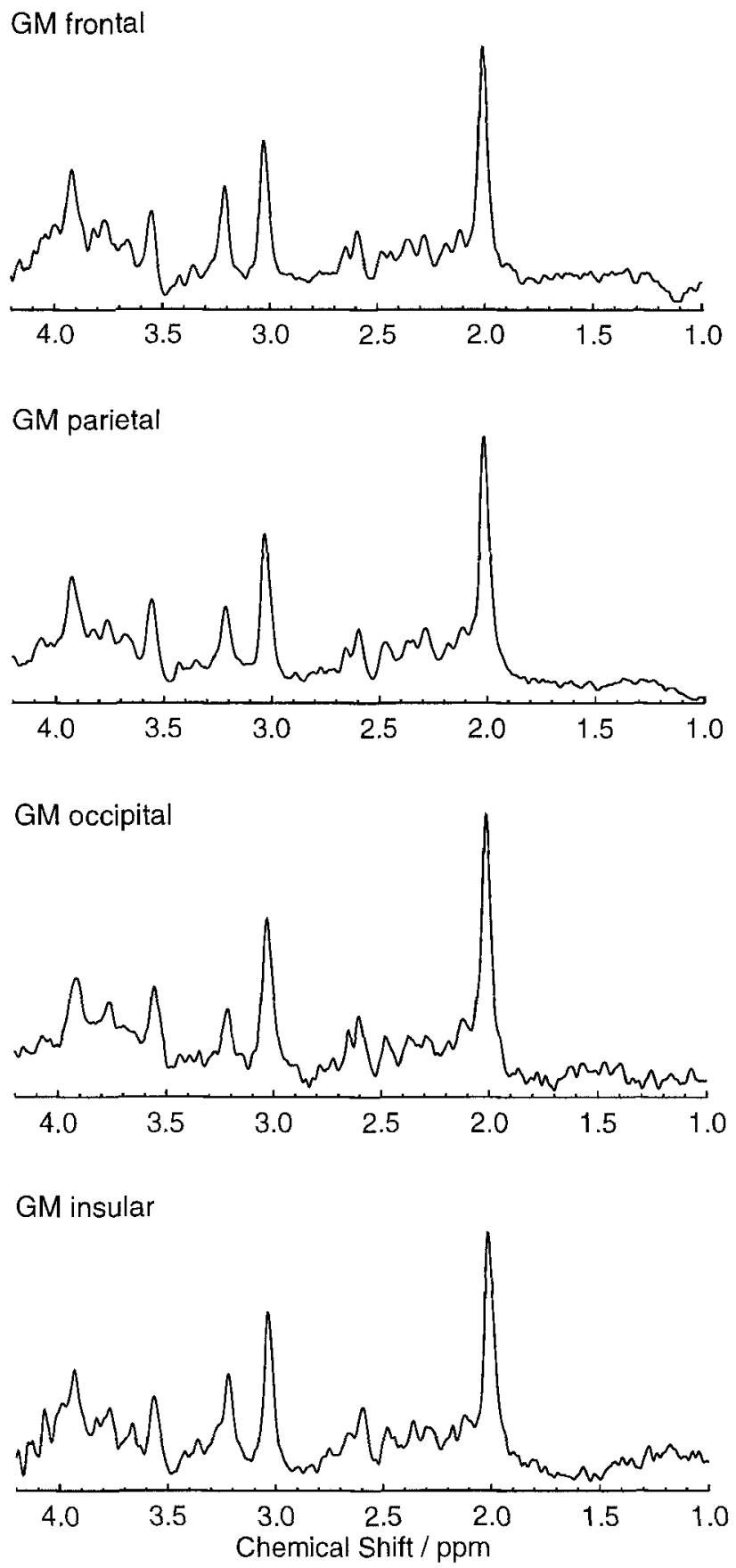

FIG. 3. Localized proton MR spectra of a 21 -year-old subject in frontal $(12 \mathrm{ml})$, parietal $(18 \mathrm{ml})$, and occipital gray matter $(8 \mathrm{ml})$, as well as of a 25 -year-old subject in the insular area $(10 \mathrm{ml})$. Other parameters as in Fig. 2.

\section{Cerebellum and Thalamus}

Spectra from the cerebellar hemisphere and vermis are shown in the upper traces of Fig. 4. Although the VOI in the cerebellar hemisphere mainly contains white matter, its metabolite composition clearly differs from that of cortical white matter (e.g., compare Fig. 2). This is even better reflected in the absolute concentrations given in Table 3. Concentrations of $\mathrm{Cr}$, Cho, and Ins are similar in both cerebellar areas but significantly higher than in any neocortical location. A prominent difference between the two cerebellar regions is the concentration of tNAA, which is significantly higher in the cerebellar hemispheres than in the central part. This is due to much higher amounts of NAAG in the hemispheres, whereas the NAA concentration in both areas is constant and slightly lower than in most cortical regions. Within the cerebellum no regional differences are observed for Glu and Gln. Whereas Glu levels are comparable to neocortical regions, Gln concentrations resemble those in gray matter and are higher than in white matter.

A representative spectrum of thalamus is shown in the bottom trace of Fig. 4. As a result of large variations in magnetic susceptibility that are mainly caused by a high iron load in adult tissues, spectra typically suffer from broad lines (i.e., a mean linewidth of $5.5 \mathrm{~Hz}$ as opposed to $3.5 \mathrm{~Hz}$ for most other brain regions at $2.0 \mathrm{~T}$ ). Still, concentrations of major metabolites can be determined very reliably as demonstrated by standard deviations that are comparable to those in other brain areas. The main differences between gray matter in cortex and thalamus are the relatively high Cho and INAA concentrations in the latter, whereas similar levels are found for $\mathrm{Cr}$ and Ins. A separate analysis of NAA and NAAG indicates the presence of high concentrations of NAAG in thalamus.

\section{Gender Effects and Hemispheric Asymmetry}

The intraindividual asymmetry parameters for Cr, tNAA, Cho, and myo-Ins are summarized in Table 4. For these metabolites no asymmetry is observed in any of the regions investigated bilaterally. In addition, no statistically significant interindividual asymmetry was detected by a $t$ test of group averages of all right- and left-hemispheric spectra.

The data base was further evaluated for gender differences. Because these assessments were performed in retrospect, the measured spectra were not equally distributed between the two sexes for all regions. However, using the data available, no statistically significant gender differences were obtained. The only exception was for Ins in parietal white matter where the ratio of femaleto-male concentrations reached $1.3(p<0.01)$, i.e., $3.4 \pm$ $0.5 \mathrm{~m} M$ for female $(n=14)$ and $2.6 \pm 0.4 \mathrm{~m} M$ for male subjects $(n=6)$.

\section{DISCUSSION}

Apart from ignoring residual $T_{2}$ and CSF corrections, the accuracy of an average concentration value as expressed by its standard deviation in Tables $1-3$, reflects the measurement precision of individual spectra (depending on SNR and VOI size), the reliability of the evaluation process (characterized by LCModel estimates of individual concentration values), and true intersubject variability. For example, for relatively large gray matter volumes with high spectral SNR, evaluation by LCModel results in individual metabolite concentrations with experimental uncertainties of only $3-7 \%$ for tNAA and $\mathrm{Cr}$, and $7-12 \%$ for Cho and Ins. The corresponding mean concentrations averaged over all subjects exhibit larger standard deviations of $6-12 \%$ for $\mathrm{tNAA}$ and $\mathrm{Cr}, 11-15 \%$ for Cho, and $15-21 \%$ for Ins (Table 2). This suggests that the 
Table 2

Metabolite Concentrations in Gray Matter $(\mathrm{mM} \pm \mathrm{SD})^{\mathrm{a}}$

\begin{tabular}{lcccc} 
& Frontal & Parietal & Occipital & Insular \\
$n=12$ & $n=19$ & $n=14$ & 16 \\
\hline Cr & $6.4 \pm 0.7$ & $6.5 \pm 0.6$ & $6.9 \pm 0.7$ & $7.0 \pm 0.6^{\star}$ \\
tNAA & $8.4 \pm 1.0$ & $8.7 \pm 0.8$ & $10.6 \pm 0.8^{\star \star \star}$ & $9.6 \pm 0.6^{\star \star}$ \\
NAA & $7.7 \pm 1.0$ & $8.2 \pm 0.8$ & $9.2 \pm 0.9^{\star \star}$ & $8.7 \pm 0.8$ \\
NAAG & $0.7 \pm 0.3$ & $0.5 \pm 0.4$ & $1.4 \pm 0.8^{\star \star}$ & $0.8 \pm 0.5^{\star}$ \\
Cho & $1.38 \pm 0.17^{\star \star *}$ & $1.10 \pm 0.14$ & $0.88 \pm 0.10^{\star \star *}$ & $1.30 \pm 0.19^{\star *}$ \\
Ins & $4.3 \pm 0.9$ & $4.3 \pm 0.7$ & $4.1 \pm 0.6$ & $4.7 \pm 0.6$ \\
Glu & $8.5 \pm 1.0$ & $8.2 \pm 1.1$ & $8.6 \pm 1.1$ & $8.8 \pm 0.8$ \\
Gln & $4.4 \pm 1.4$ & $3.8 \pm 1.4$ & $3.9 \pm 1.1$ & $4.9 \pm 1.6^{*}$ \\
FWHM & $3.51 \pm 0.34$ & $3.50 \pm 0.33$ & $4.06 \pm 0.31$ & $4.06 \pm 0.43$ \\
\hline
\end{tabular}

a $\mathrm{mM}$ refers to $\mathrm{mmol} /$ liter $\mathrm{VOI}$.

b FWHM: full linewidth at half maximum $(\mathrm{Hz})$ of the $\mathrm{Cr}$ signal at $3.03 \mathrm{ppm}$.

${ }^{* * *} p<0.001,{ }^{* *} p<0.01,{ }^{*} p<0.05$ for comparisons to parietal gray matter.

interindividual variability due to small differences in VOI location and/or true physiologic differences is larger than the inaccuracy of the MRS quantification method. Similar arguments hold true for white matter, although smaller volumes and correspondingly lower SNR yield

\section{Cerebellum hemisphere}

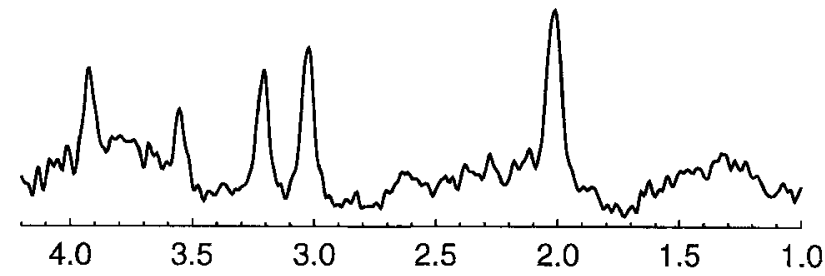

\section{Cerebellum central}

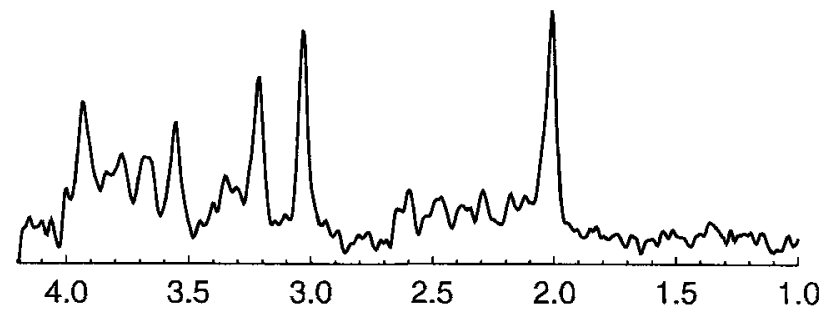

Thalamus

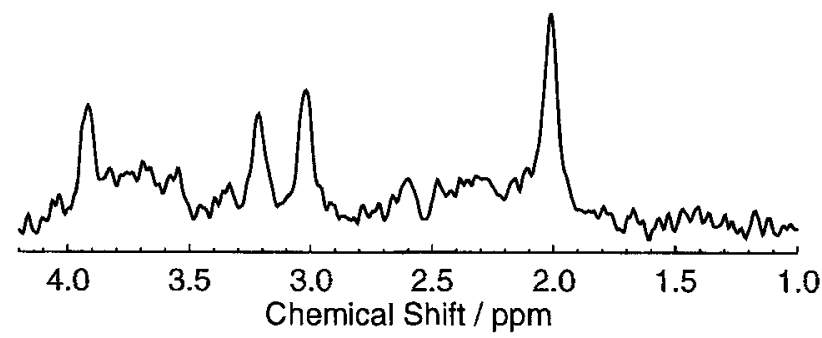

FIG. 4. Localized proton MR spectra of a 25 -year-old subject in the cerebellar hemisphere $(8 \mathrm{ml})$, of a 25 -year-old subject in the central cerebellum $(8 \mathrm{ml})$, and of an 18-year-old subject in thalamus $(6.4 \mathrm{ml})$. Other parameters as in Fig. 2. slightly larger experimental uncertainties for individual concentrations.

It is also possible that the presence of intersubject variability precludes the detection of small differences between group averages of right and left hemispheres or between females and males. However, with the present accuracy (Table 4), our data do not support concepts of metabolic laterality previously hypothesized not only for the temporal lobe $(20,21)$, but also for parietal and occipital lobes (21). Pertinent reports are based on ratios of resonance intensities from long-echo time recordings without absolute quantification. Conversely, a long-echo time spectroscopic imaging study is in line with our finding of metabolic hemispheric symmetry (8).

In general, the present work confirms the absence of a gender difference. This particularly applies to major metabolites such as tNAA and $\mathrm{Cr}$ with the best experimental accuracy. After ruling out that artifacts contribute to the exceptional result of a 1.3-fold higher Ins concentration in parietal white matter of female subjects, we fail to report an explanation.

\section{NAA and NAAG}

The finding of a heterogeneous distribution of tNAA in human brain is supported by studies in which no distinction between NAA and NAAG has been made. The frontal to parieto-occipital increase of tNAA has been described $(6,7)$, as well as the generally higher tNAA concentration in white matter as compared to gray matter $(3,4,8,9)$. A relatively higher tNAA level in the cerebellar hemisphere as compared to the vermis has also been detected by spectroscopic imaging (22).

Extending these observations by separating NAA and NAAG demonstrates that the significant differences in tNAA within and between different brain tissues are mainly due to a corresponding regional variation of NAAG. The distribution of NAA is rather homogeneous, with the exception of a slightly, but significantly higher concentration in occipital gray matter, i.e., in the functional area of the visual cortex. These findings parallel neuroanatomic reports of a constant neuronal cell density in the neocortex except for a higher density exclusively in primary visual cortex $(23,24)$. It further supports the use of NAA as a neuronal marker but also 
Table 3

Metabolite Concentrations in Cerebellum and Thalamus $(\mathrm{mM} \pm \mathrm{SD})^{\mathrm{a}}$

\begin{tabular}{lccc}
\hline & \multicolumn{2}{c}{ Cerebellum } & Thalamus \\
\cline { 2 - 3 } & $\begin{array}{c}\text { Hemisphere } \\
\mathrm{n}=19\end{array}$ & $\begin{array}{c}\text { Vermis } \\
\mathrm{n}=8\end{array}$ & $\mathrm{n}=16$ \\
\hline Cr & $8.7 \pm 1.3$ & $9.0 \pm 0.9$ & $6.8 \pm 0.7$ \\
tNAA & $11.0 \pm 0.8$ & $8.7 \pm 0.9^{\star \star \star}$ & $10.5 \pm 0.7$ \\
NAA & $7.4 \pm 1.0$ & $7.4 \pm 0.8$ & $7.3 \pm 1.3$ \\
NAAG & $3.6 \pm 0.9$ & $1.3 \pm 0.8^{\star \star \star}$ & $3.2 \pm 1.1$ \\
Cho & $2.21 \pm 0.29$ & $2.19 \pm 0.30$ & $1.81 \pm 0.21$ \\
Ins & $5.8 \pm 1.1$ & $5.6 \pm 1.1$ & $3.6 \pm 0.9$ \\
Glu & $7.7 \pm 2.5$ & $7.5 \pm 1.4$ & $6.9 \pm 2.2$ \\
Gln & $4.3 \pm 2.5$ & $5.4 \pm 2.2$ & $2.9 \pm 1.8$ \\
FWHM & $5.03 \pm 0.74$ & $3.72 \pm 0.47$ & $5.48 \pm 0.69$ \\
\hline
\end{tabular}

a $\mathrm{mM}$ refers to $\mathrm{mmol} /$ liter $\mathrm{VOI}$.

${ }^{b}$ FWHM: full linewidth at half maximum $(\mathrm{Hz})$ of the $\mathrm{Cr}$ signal at $3.03 \mathrm{ppm}$.

${ }^{* \star \star} p<0.001$ for comparisons between both cerebellar regions.

precludes that this compound has a (metabolic) function that is specific for a certain cortical area.

The concentration of NAAG varies from $0.5 \mathrm{mM}$ in parietal gray matter to $3.6 \mathrm{mM}$ in the cerebellar hemisphere. This distribution appears to be determined by both a general caudal increase proceeding from cerebrum to spinal cord (25) and a large difference in NAAG content between gray and white matter. Because NAAG like NAA is a neuronal compound (25-27), it shares the apparent discrepancy to be highly concentrated not only in cell bodies (gray matter) but also in myelinated axons (white matter). A similar conclusion was drawn from an in vitro study of metabolites in rat brain, which showed high levels of NAA and especially NAAG in the adult optic nerve (28). An independent confirmation was given by immunohistochemistry demonstrating NAA and NAAG immunoreactivity in all parts of the nerve cells including dendrites, axons, and synaptic terminals (29).

Whereas spectra from cortex and the cerebellar vermis show mean resonance linewidths of $3.3-4.1 \mathrm{~Hz}$, the cerebellar hemispheres and thalamus result in mean linewidths of $5.0-5.5 \mathrm{~Hz}$ (Tables 1-3). Although the accuracy of LCModel depends on the magnetic field homogeneity and corresponding spectral resolution (30), the standard deviations for NAAG levels in these regions are similar to those in other regions. This suggests that the high NAAG concentrations found in thalamus and cerebellar hemisphere are reliable. It is further supported by the observation of correspondingly high NAAG levels in thalamus of young children where spectral resolution is excellent. Although cortical NAAG concentrations are low at birth and increase only slowly, thalamus concentrations are much higher (unpublished results).

\section{Total Creatine}

The concentration of $\mathrm{Cr}$ is rather constant within each tissue type, but increases significantly from cortical white matter $(5.5-5.7 \mathrm{mM})$ to gray matter $(6.4-7.0 \mathrm{mM})$ and cerebellum $(8.7-9.0 \mathrm{mM})$. In thalamus the concentration $(6.8 \mathrm{mM})$ is similar to that in cortical gray matter. Higher Cr levels in gray matter than in white matter have also been found in previous single-voxel MRS studies ( 3 , 4). In contrast, the usually much larger standard deviations for (relaxation-weighted) metabolite levels from spectroscopic imaging have precluded the observation of a statistically significant difference for Cr in white and gray matter $(8,9)$. One study indicated higher $\mathrm{Cr}$ levels in gray matter when using image segmentation (10). Of course, this latter approach is applicable to metabolites with a homogeneous distribution within the same tissue type. For Cr it leads to similar results as obtained with single-voxel techniques.

The high concentration of total creatine in the cerebellum is in agreement with previous reports for both a hemispheric location (6) and the vermis $(3,8)$. In addition, it corresponds to high amounts of cytosolic and mitochondrial creatine kinase (CK) isoenzymes in this brain region $(31,32$, and references therein). CK catalyzes the reversible phosphate transfer from $\mathrm{PCr}$ and $\mathrm{ADP}$ to $\mathrm{Cr}$ and ATP. Its cellular distribution reflects the heterogeneity of brain ATP metabolism (33). High levels of CK in the granular layer of the cerebellum, which is rich in mitochondria, are probably coupled to a high energy demand of synaptic and glial-neuron interactions in this region (31). Moreover, in chicken the cerebellum has been demonstrated as the only brain region with a muscle type CK, a finding that has tentatively been ascribed to the energy demands of the cerebellar Purkinje neurons (31).

\section{Choline-Containing Compounds}

Of all metabolites but NAAG, Cho shows the largest regional variability in the brain. Highly significant differences are found between cortical gray and white matter, thalamus, and cerebellum with concentrations increasing in that order. Confirming previous studies using both single-voxel MRS $(3,4,6)$ and spectroscopic imaging (8, 9), cortical gray matter contains the lowest Cho concentrations, whereas cerebellar levels are by a factor of 2-2.5 higher. In addition to these differences between tissues, there exists a strong caudal gradient of decreasing Cho in

Table 4

Intraindividual Right-to-left Concentration Ratios ( $\pm \mathrm{SD}$ )

\begin{tabular}{lcccccc}
\hline & $\begin{array}{c}\text { Frontal } \\
\mathrm{n}=7^{\mathrm{a}}\end{array}$ & $\begin{array}{c}\text { White matter } \\
\text { parietal } \\
\mathrm{n}=8\end{array}$ & $\begin{array}{c}\text { Occipital } \\
\mathrm{n}=10\end{array}$ & $\begin{array}{c}\text { Gray matter } \\
\text { insular } \\
\mathrm{n}=8\end{array}$ & $\begin{array}{c}\text { Cerebellum } \\
\text { hemisphere } \\
\mathrm{n}=8\end{array}$ & $\begin{array}{c}\text { Thalamus } \\
n=6\end{array}$ \\
\hline Cr & $1.08 \pm 0.10$ & $1.09 \pm 0.14$ & $1.00 \pm 0.19$ & $0.98 \pm 0.11$ & $1.01 \pm 0.19$ & $1.01 \pm 0.09$ \\
tNAA & $1.10 \pm 0.11$ & $1.05 \pm 0.11$ & $1.00 \pm 0.04$ & $1.01 \pm 0.11$ & $1.03 \pm 0.09$ & $1.02 \pm 0.09$ \\
Cho & $0.90 \pm 0.12$ & $1.09 \pm 0.19$ & $0.97 \pm 0.13$ & $0.96 \pm 0.14$ & $1.10 \pm 0.21$ & $1.09 \pm 0.13$ \\
Ins & $1.02 \pm 0.37$ & $1.11 \pm 0.19$ & $1.04 \pm 0.21$ & $1.03 \pm 0.14$ & $1.07 \pm 0.18$ & $1.19 \pm 0.31$ \\
\hline
\end{tabular}

${ }^{a}$ Number of subjects with bilateral spectral acquisitions. 
cortical gray matter. This finding is in good qualitative agreement with a determination of choline-containing compounds in an autopsy study yielding $12.8,10.5$, and $7.8 \mathrm{nmol} / \mathrm{mg}$ protein in frontal, parietal, and occipital human cortex, respectively (34). It is further in line with two recent spectroscopic imaging studies of elderly controls in which the intensity ratio of $\mathrm{Cho} / \mathrm{Cr}$ resonances decreased (35) and that of NAA/Cho increased $(35,36)$ from frontal to posterior paramedian gray matter.

It may be argued that the observed Cho gradient in gray matter is partly due to different partial volume effects with white matter showing higher Cho concentrations. However, visual inspection of gray matter volumes shows that the VOIs contain only minor and similar portions of white matter. It is therefore more likely that the Cho distribution in gray matter as well as in other brain regions is correlated to regional differences in membrane lipid composition and metabolism.

The main metabolites that contribute to the protondetected Cho resonance in vivo are PCh and GPC. These compounds are involved in the metabolism of the membrane lipids phosphatidylcholine (i.e., lecithin) and ceramidephosphocholine (i.e., sphingomyelin) $(37,38)$. Due to its high myelin content, white matter contains more lipids than gray matter $(15.6 \%$ versus $5.9 \%$ of total wet weight) and also shows a different lipid composition. Under physiologic conditions both lecithin and sphingomyelin are subject to continuous breakdown and resynthesis. Although glycerophospholipids are more stable in myelin than in other membranes with turnover rates between 1 week and 1 month (39), such rates also depend on the lipid side-chain composition which in turn exhibits regional variations $(37,38)$. Because PCh and GPC are part of the metabolic pathway of lecithin and sphingomyelin, the concentration of Cho and the content of the choline-containing phospholipids in a certain brain region will be roughly correlated. However, they cannot be assumed to be directly proportional due to the many interfering variables.

\section{Myo-Inositol}

The concentration of Ins is lowest in white matter and thalamus, slightly higher in cortical gray matter $(3,4)$, and highest in both regions of the cerebellum. The latter observation also applies to $\mathrm{Cr}$ and Cho. Based on studies of cell type-specific cultures, Ins may be considered as a glial marker that is not present in neurons (40). Apart from its role in the metabolism of membrane-bound inositol phospholipids and their links to a number of neuronal signaling systems, Ins mainly serves as an important brain osmolyte.

Because Ins is involved in many biochemical processes, the reason for its heterogeneity in brain remains speculative. For example, the high levels of Ins (and Cho) in the cerebellum might be due to a higher glial and/or membrane density in this region. Alternatively, cerebellar osmoregulation may require high Ins levels as recent reports have mentioned an enhanced expression of the sodium myo-inositol cotransporter mRNA during hypernatremia especially in cerebellum $(41,42)$. During changes in osmolality this cotransporter is involved in a redistribution of intracellular and extracellular Ins.

\section{Glutamate and Glutamine}

Both Glu and Gln show a relatively homogeneous distribution within single tissue types. In gray matter the accuracy of the observed concentrations is higher than in white matter due to larger VOI sizes and correspondingly higher SNR. Despite substantial overlap of spectral resonances, LCModel allows a reliable separation of Glu and Gln contributions at 2.0 T. This is underlined by the fact that for both Glu and Gln the difference between concentrations in cortical gray inatter $(8.4 \pm 1.0$ and $4.0 \pm 1.3$ $\mathrm{m} M$, respectively) and white matter $(6.5 \pm 1.9$ and $1.9 \pm$ $1.5 \mathrm{mM}$, respectively) is highly significant ( $p<0.0001)$.

The present concentration values correlate well with a previous determination of total Glu and Gln of 12.5 $\mathrm{mmol} / \mathrm{kg}$ wet weight in gray matter and $8.1 \mathrm{mmol} / \mathrm{kg}$ in white matter (3). Qualitatively, a recent spectroscopic imaging study of Glu also indicated a higher level in gray matter than in white matter (43). Despite potential complications due to postmortem proteolysis, autopsy studies yielded Glu concentrations of $7-10 \mathrm{mmol} / \mathrm{kg}$ for cortical and cerebellar gray matter and $4-5 \mathrm{mmol} / \mathrm{kg}$ for white matter, as well as Gln concentrations of 4-5 $\mathrm{mmol} / \mathrm{kg}$ for cortical gray matter with similar or higher levels in cerebellar cortex (44, and references therein).

\section{CONCLUDING REMARKS}

MRS strategies that optimize the experimental conditions for absolute quantification allow an objective and reproducible determination of metabolite concentrations within the human brain. In this context it is worth noting that single-voxel MRS features such as the ability to use long repetition times and the achievable spectral quality do not represent academic advantages, but are crucial in translating "spectral changes" into meaningful biochemical quantities. In particular, absolute metabolite concentrations guarantee reliable intrasubject (e.g., follow-up studies) and intersubject comparisons (e.g., patient versus control).

Extending previous observations this study unraveled detailed regional differences in cerebral metabolite patterns between as well as within tissue types. The data serve as base material for a variety of metabolic and functional brain studies and are already used as control for metabolic examinations of patients with brain disorders.

\section{ACKNOWLEDGMENTS}

The authors thank Wolfgang Hänicke and Dr. Stephen Provencher for helpful discussions about spectral data evaluation and Xiangling Mao for her development of spectral processing software.

\section{REFERENCES}

1. J. Frahm, H. Bruhn, M. L. Gyngell, K.-D. Merboldt, W. Hänicke, R. Sauter, Localized proton NMR spectroscopy in different regions of 
the human brain in vivo: relaxation times and concentrations of cerebral metabolites. Magn. Reson. Med. 11, 47-63 (1989).

2. P. A. Narayana, L. K. Fotedar, E. F. Jackson, T. P. Bohan, I. J. Butler, J. S. Wolinsky, Regional in vivo proton magnetic resonance spectroscopy of brain. J. Magn. Reson. 83, 44-52 (1989).

3. T. Michaelis, K.-D. Merboldt, H. Bruhn, W. Hänicke, J. Frahm, Absolute concentrations of metabolites in the adult human brain in vivo: quantification of localized proton MR spectra. Radiology 187, 219227 (1993)

4. R. Kreis, T. Ernst, B. D. Ross, Absolute quantitation of water and metabolites in the human brain. II. Metabolite concentrations. J. Magn. Reson. B Series 102, 9-19 (1993).

5. P. J. W. Pouwels, W. Hänicke, J. Frahm, On a concentration gradient of cerebral metabolites in human gray matter as determined by quantitative localized proton MRS, in "Proc., SMR, 3rd Annual Meeting, Nice, 1995," p. 1957.

6. J. Hennig, H. Pfister, T. Ernst, D. Ott, Direct absolute quantification of metabolites in the human brain with in vivo localized proton spectroscopy. NMR Biomed. 5, 193-199 (1992).

7. P. Christiansen, P. Toft, H. B. W. Larsson, M. Stubgaard, O. Henriksen, The concentration of $N$-acetylaspartate, creatine and phosphocreatine, and choline in different parts of the brain in adulthood and senium. Magn. Reson. Imaging 11, 799-806 (1993).

8. G. Tedeschi, A. Bertolino, A. Righini, G. Campbell, R. Raman, J. H, Duyn, C. T. W. Moonen, J. R. Alger, G. Di Chiro, Brain regional distribution pattern of metabolite signal intensities in young adults by proton magnetic resonance spectroscopic imaging. Neurology $\mathbf{4 5}$, 1384-1391 (1995).

9. B. J. Soher, P. C. M. van Zijl, J. H. Duyn, P. B. Barker, Quantitative proton MR spectroscopic imaging of the human brain. Magn. Reson. Med. 35, 356-363 (1996).

10. H. P. Hetherington, J. W. Pan, G. F. Mason, D. Adams, M. J. Vaughn, D. B. Twieg, G. M. Pohost, Quantitative ${ }^{1} \mathrm{H}$ spectroscopic imaging of human brain at $4.1 \mathrm{~T}$ using image segmentation. Magn. Reson. Med. 36, 21-29 (1996).

11. J. Frahm, T. Michaelis, K.-D. Merboldt, H. Bruhn, M. L. Gyngell, W. Hänicke, Improvements in localized ${ }^{2}$ II NMR spectroscopy of human brain: water suppression, short echo times and $1 \mathrm{ml}$ resolution. J. Magn. Reson. 90, 464-473 (1990).

12. U. Klose, In vivo proton spectroscopy in presence of eddy currents. Magn. Reson. Med. 14, $26-30$ (1990).

13. S. W. Provencher, Estimation of metabolite concentrations from localized in vivo proton NMR spectra. Magn. Reson. Med. 30, 672-679 (1993).

14. P. J. W. Pouwels, J. Frahm, Differential distribution of NAA and NAAG in human brain as determined by localized proton MRS. NMR Biomed. 10, 73-78 (1997).

15. K.-D. Merboldt, D. Chien, W. Hänicke, M. L. Gyngell, H. Bruhn, J. Frahm, Localized ${ }^{31} \mathrm{P}$ NMR spectroscopy of the adult human brain in vivo using stimulated-echo (STEAM) sequences. J. Magn. Reson. 89, 343-361 (1990).

16. P. B. Barker, S. N. Breiter, B. J. Soher, J. C. Chatham, J. R. Forder, M. A. Samphilipo, C. A. Magee, J. H. Anderson, Quantitative proton spectroscopy of canine brain: in vivo and in vitro correlations. Magn, Reson. Med. 32, 157-163 (1994).

17. M. Ala-Korpela, P. Posio, S. Mattila, A. Korhonen, S. R. Williams, Absolute quantification of phospholipid metabolites in brain-tissue extracts by 'H NMR spectroscopy. J. Magn. Reson. Series B 113, 184-189 (1996).

18. K. L. Behar, D. L. Rothman, D. D. Spencer, O. A. C. Petroff, Analysis of macromolecule resonances in ${ }^{1} \mathrm{H}$ NMR spectra of human brain. Magn. Reson. Med. 32, 294-302 (1994).

19. R. A. Kauppinen, T. Niskanen, J. Hakumaki, S. R. Williams, Quantitative analysis of ${ }^{1} \mathrm{H}$ NMR detected proteins in the rat cerebral cortex in vivo and in vitro. NMR Biomed. 6, 242-247 (1993).

20. D. Bernard, P. M. Walker, N. Baudouin-Poisson, M. Giroud, H. Fayolle, R. Dumas, D. Martin, D. Binnert, F. Brunotte, Asymmetric metabolic profile in mesial temporal lobes: localized H-1 MR spectroscopy in healthy right-handed and non-right-handed subjects. Radiology 199, 381-389 (1996).

21. R. Jayasundar, P. Raghunathan, Evidence for lateralization of metabolites from the proton MRS of brain, in "Proc., ISMRM, 4th Annual Meeting, New York, 1996," p. 955.

22. M. J. Fulham, M. J. Dietz, J. H. Duyn, H. H.-L. Shih, J. R. Alger, G. Di Chiro, Transsynaptic reduction in $N$-acetylaspartate in cerebellar di- aschisis: a proton MR spectroscopic imaging study. J. Comput. Assist. Tomogr. 18, 697-704 (1994).

23. A. J. Rockel, R. W. Hiorns, T. P. S. Powell, The basic uniformity in structure of the neocortex. Brain 103, 221-244 (1980).

24. G. Leuba, L. J. Garey, Comparison of neuronal and glial numerical density in primary and secondary visual cortex of man. Exp. Brain Res. 77, 31-38 (1989).

25. R. D. Blakely, J. T. Coyle, in "International Review of Neurobiology" (J. R. Smythies, R. J. Bradley, Eds.), vol. 30, pp. 39-100, Academic Press, San Diego, 1988.

26. D. L. Birken, W. H. Oldendorf, N-Acetyl-L-aspartic acid: a literature review of a compound prominent in ${ }^{1} \mathrm{H}$ NMR spectroscopic studies of brain. Neurosci. Biobehav. Rev. 13, 23-31 (1989).

27. J. R. Moffett, M. A. A. Namboodiri, Differential distribution of $N$ acetylaspartylglutamate and $N$-acetylaspartate immunoreactivities in rat forebrain. J. Neurocytol. 24, 409-433 (1995).

28. C.-L. Florian, S. R. Williams, K. K. Bhakoo, M. D. Noble, Regional and developmental variations in metabolite concentration in the rat brain and eye: a study using ${ }^{1} \mathrm{H}$ NMR spectroscopy and high performance liquid chromatography, Neurochem. Res. 21, 1065-1074 (1996).

29. J. R. Moffett, M. A. A. Namboodiri, J. H. Neale, Enhanced carbodiimide fixation for immunohistochemistry: application to the comparative distributions of $N$-acetylaspartylglutamate and $N$-acetylaspartate immunoreactivities in rat brain. J. Histochem. Cytochem. 41, 559-570 (1993).

30. S. W. Provencher, W. Hänicke, T. Michaelis, Automated quantitation of localized ${ }^{1} \mathrm{H}$ MR spectra in vivo: capabilities and limitations, in "Proc., SMR, 3rd Annual Meeting, Nice, 1995," p. 1952.

31. P. Kaldis, W. Hemmer, E. Zanolla, D. Holtzman, T. Wallimann, 'Hot spots' of creatine kinase localization in brain: cerebellum, hippocampus and choroid plexus. Dev. Neurosci. 18, 542-554 (1996).

32. S. E. Ilyin, G. Sonti, G. Molloy, C. R. Plata-Salaman, Creatine kinase-B mRNA levels in brain regions from male and female rats. Mol. Brain Res. 41, 50-56 (1996).

33. D. Holtzman, Brain creatine kinases and phosphocreatine: an update. Dev. Neurosci. 18, 522-523 (1996).

34. R. M. Nitsch, J. K. Blusztajn, F. M. Doyle, Y. Robitaille, R. J. Wurtman, J. H. Growdon, S. J. Kish, Phospholipid metabolite levels are altered in cerebral cortex of patients with dominantly inherited olivopontocerebellar atrophy. Neurosci. Lett. 161, 191-194 (1993).

35. G. Tedeschi, A. Bertolino, N. Lundbom, S. Bonavita, N. J. Patronas, J. H. Duyn, L. Verhagen Metman, T. N. Chase, G. Di Chiro, Cortical and subcortical chemical pathology in Alzheimer's disease as assessed by multislice proton magnetic resonance spectroscopic imaging. Neurology 47, 696-704 (1996).

36. S. MacKay, D. J. Meyerhoff, J. M. Constans, D. Norman, G. Fein, M. W. Weiner, Regional gray and white matter metabolite differences in subjects with $A D$, with subcortical ischemic vascular dementia, and elderly controls with ${ }^{1} \mathrm{H}$ magnetic resonance spectroscopic imaging. Arch. Neurol. 53, 167-174 (1996).

37. B. W. Agranoff, A. K. Hajra, Lipids, in "Basic Neurochemistry: Molecular, Cellular, and Medical Aspects" (G.J. Siegel, B.W. Agranoff, R.W. Albers, P.B. Molinoff, Eds.), 5th ed., pp. 97-116, Raven Press, New York, 1994.

38. P. S. Sastry, Lipids of nervous tissue: composition and metabolism. Prog. Lipid Res. 24, 69-176 (1985).

39. P. Morell, A. H. Ousley, Metabolic turnover of myelin glycerophospholipids. Neurochem. Res. 19, 967-974 (1994).

40. A. Brand, C. Richter-Landsberg, D. Leibfritz, Multinuclear NMR studies on the energy metabolism of glial and neuronal cells. Dev. Neurosci. 15, 289-298 (1993).

41. Y. Minami, K. Inoue, S. Shimada, H. Morimura, A. Miyai, A. Yamauchi, T. Matsunaga, M. Tohyama, Rapid and transient up-regulation of $\mathrm{Na}^{+} / \mathrm{myo}$-inositol cotransporter transcription in the brain of acute hypernatremic rats. Mol. Brain Res. 40, 64-70 (1996).

42. L. Ibsen, K. Strange, In situ localization and osmotic regulation of the $\mathrm{Na}^{+}$-myo-inositol cotransporter in rat brain. Am. J. Physiol. 271, F877-885 (1996).

43. J. W. Pan, G. F. Mason, G. M. Pohost, H. P. Hetherington, Spectroscopic imaging of human brain glutamate by water-suppressed Jrefocused coherence transfer at 4.1 T. Magn. Reson. Med. 36, 7-12 (1996).

44. R. Knörle, D. Assmann, G. B. Landwehrmeyer, R. Scheremet, K. Müller, T. J. Feuerstein, Aspartate, glutamate, glutamine, glycine and $\gamma$-aminobutyric acid in human bioptic neocortical areas: comparison to autoptic tissue. Neurosci. Lett. 221, 169-172 (1997). 